\title{
DACH-Platin Micelle NC-4016
}

National Cancer Institute

\section{Source}

National Cancer Institute. DACH-Platin Micelle NC-4016. NCI Thesaurus. Code C113175.

Polymeric micellar nanoparticles containing diaminocyclohexane platinum (DACH-platin or DACH-Pt) with potential antineoplastic activity. DACH-platin micelle NC-4016 is prepared through the formation of a polymer-metal complex between DACH-platin and the polyethylene glycol-poly (glutamic acid) block copolymer, PEG-P(Glu). DACH-platin, an active metabolite of the platinum-based antineoplastic agent oxaliplatin, is highly hydrophobic and toxic when administered systemically. The use of polymeric micelles incorporating DACH-platin may both increase cell permeability and enhance the retention of the agent. This allows an extended half-life in the blood circulation and a selective and high accumulation of DACH-platin at tumor sites. This results in increased anticancer efficacy while reducing side effects due to DACH-platin toxicity. Upon intravenous administration and internalization by tumor cells, DACH-platin binds to and causes both inter- and intra-strand cross-links in DNA, forming platinum adducts and triggering tumor cell apoptosis. 